\title{
A Rare Case of Spinal Sarcoidosis Presenting as Multiple Bone Marrow Oedematous Lesions
}

\author{
Anat Bel-ange ${ }^{1}$, Sigal Tal ${ }^{2}$, Micha Rapoport ${ }^{1}$ \\ ${ }^{1}$ Department of Internal Medicine "C", Assaf Harofeh Medical Center, affiliated to Sackler Medical School, Tel Aviv University, Zerifin, Israel \\ ${ }^{2}$ Department of Radiology, Assaf Harofeh Medical Center, affiliated to Sackler Medical School, Tel Aviv University, Zerifin, Israel
}

How to cite this article: Bel-ange A, Tal S, Rapoport M. A rare case of spinal sarcoidosis presenting as multiple bone marrow oedematous lesions. EJCRIM 2018;5: doi:10.12890/2018_000907.

Conflicts of Interests: The Authors declare that there are no competing interests.

This article is licensed under a Commons Attribution Non-Commercial 4.0 License

\section{ABSTRACT}

Sarcoidosis is a systemic disorder that most commonly affects the lungs. Bone involvement is rare, and spinal involvement is even more rare. The presence of focal lesions of the vertebrae is highly suspicious of advanced malignancy. However, malignant metastatic spread to the spine involves the vertebral cortex rather than the bone marrow itself, a distinction that is often missed and therefore misleading. We describe here a middle-aged woman with multiple focal oedematous lesions of the bone marrow suspected of being advanced malignancy but finally diagnosed as a rare case of spinal sarcoidosis.

\section{LEARNING POINTS}

- This article describes a rare case of vertebral sarcoidosis.

- It emphasizes the often-missed distinction between bone marrow and cortical lesions in the diagnostic work-up of focal spinal lesions.

- It also underscores the combined use of MRI together with FDG/PET-CT in elucidating the nature of lesions confined to the bone marrow.

\section{KEYWORDS}

Sarcoidosis, sarcoid bone lesions, spine, MRI, bone marrow oedema

\section{CASE DESCRIPTION}

A 55-year-old woman with a past medical history of right congenital hip dysplasia, Erb's palsy and a recent diagnosis of psoriasis, was referred to our internal medicine department with increasing right leg pain and abnormal findings on spinal MRI described as multifocal lesions with decreased T1-T2 signal, with enhancement after gadolinium administration (Fig. 1). There was no family or personal history of cancer, fever and/or or recent weight loss. Physical examination was normal apart from partial restriction of the right hip joint.

Extensive work-up for a possible primary malignancy including blood count and chemistry, protein electrophoresis, bone marrow aspiration, mammography, upper and lower endoscopic GI studies, bone and thyroid scan, chest and abdominal CT scan, abdominal and pelvic US and gynaecological examination, did not reveal any malignancy. Levels of anti-nuclear and rheumatoid factors and antibodies to cANCA and pANCA were within the normal range and HLA B27 was not detected. An FDG PET/CT scan showed pathological hypermetabolic uptake in the vertebrae. This was followed by a biopsy from the L4 vertebra which revealed prominent intramedullary fibrosis and a non-necrotizing granulomatous reaction characteristic of sarcoidosis, but no evidence of mycobacterium or fungal infection. Serum angiotensin-converting enzyme (ACE) levels were elevated above the normal range. 
A diagnosis of sarcoidosis was made and systemic steroid therapy was initiated, resulting in complete disappearance of all spinal pathological uptake as demonstrated by a normal repeat FDG PET/CT scan performed 5 months after discharge. Steroid treatment was halted after the patient developed avascular necrosis of the femur. The 1-year follow-up after steroid cessation revealed elevated serum ACE levels and the reappearance of pathological findings on a spinal FDG PET/CT scan.

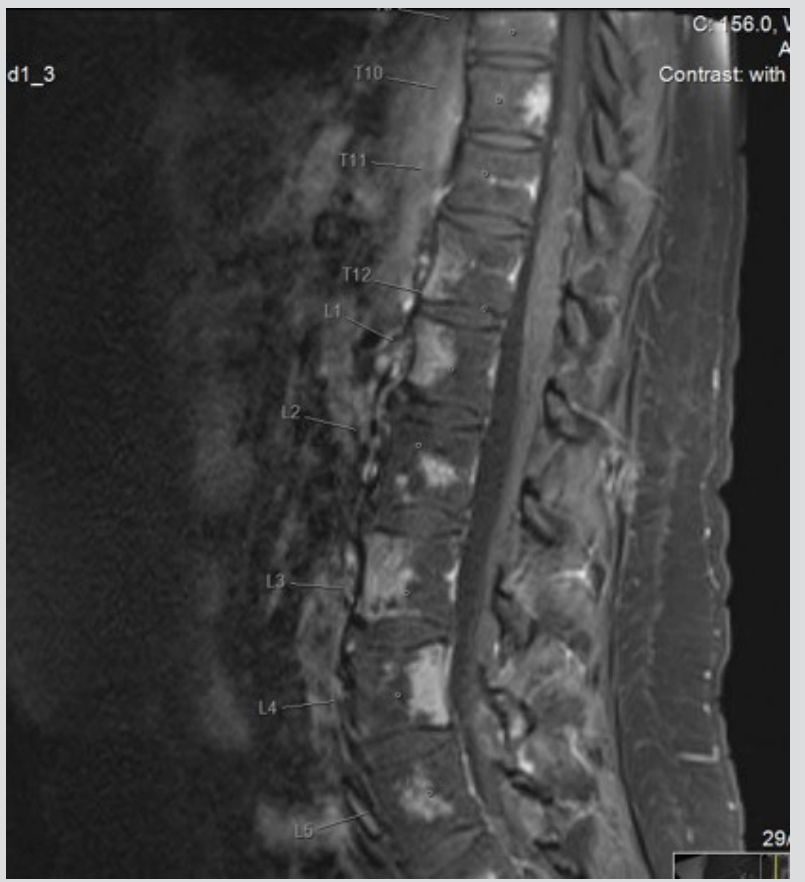

Figure 1. Abnormal findings on spinal MRI

\section{DISCUSSION}

The initial and quite rare presentation of sarcoidosis in this patient was the presence of focal oedematous lesions in the spinal bone marrow which were detected only by an MRI scan. Bone marrow oedema presents as excessive water signals in the marrow space on MRI. In most cases, histological analyses of these lesions demonstrate fibrosis, increased vascularization and lymphocytic infiltrates rather than oedematous changes ${ }^{[1]}$. The differential diagnosis of spinal focal lesions is very broad and the presence of spinal skeletal lytic or osteoblastic findings on plain radiographs or a CT scan is highly suspicious of metastatic spread and commonly triggers an extensive search for a primary malignancy. However, the ability of a CT scan to detect either the more common lytic or less common osteoblastic bone metastases is limited to the cortical bone as focal bone marrow lesions are not detected. In contrast to a CT scan, the MRI technique can detect diffuse and/or focal pathologies in the bone marrow, whether these pathologies remain restricted to the bone marrow or progress later to destruction of the cortical bone as happens in advanced metastatic spread to the skeleton ${ }^{[2,3]}$. The differential diagnosis for focal bone marrow oedematous lesions on MRI is extremely broad ${ }^{[1,4]}$. The number of potential pathologies can be significantly reduced when only entities with a low T1 signal intensity, as happened in our patient, are considered and includes among others: haematopoietic and solid malignancies, metabolic diseases, spondyloarthropathies, infectious and granulomatous diseases such as sarcoidosis, renal osteodystrophy, and miscellaneous diseases such as myelofibrosis, mastocytosis, haemosiderosis, lipid storage diseases and gout ${ }^{[4]}$. In our patient, most of these pathologies were ruled out on clinical grounds and/or the absence of typical serology. Nevertheless, a CT-guided bone marrow aspiration biopsy was mandatory to completely rule out malignancy and establish the diagnosis of vertebral sarcoidosis.

It should be noted that extra-pulmonary sarcoidosis of the bone is a very rare diagnosis, and more so as an initial presentation. Case reports of spinal sarcoidosis have been reported since the $1950 \mathrm{~s}^{[5-11]}$. However, in all of these reports the first presentation was systemic disease affecting multiple organs rather than bone involvement. Obviously, advanced imaging modalities such as MRI were not used in many of the older reports and it remains unknown if and when involvement of bone marrow did occur. Thus, our case is unique in that it is the first description of bone marrow focal oedematous lesions due to sarcoidosis restricted to the bone marrow alone as a first manifestation, without any other organ involvement. 
It is also of interest that FDG-PET/CT is highly sensitive for detecting granulomatous bone marrow infiltration ${ }^{[12,13]}$. This modality being increasingly used as a diagnostic tool that strengthens the diagnosis of sarcoidosis and for follow-up after treatment.

In conclusion, this rare case of vertebral sarcoidosis emphasizes the often missed but very significant distinction between bone marrow and cortical lesions in the diagnostic work-up of focal spinal lesions. It also underscores the combined use of MRI together with FDG/PET$\mathrm{CT}$ in elucidating the nature of lesions confined to the bone marrow. Finally, the diagnosis of bone sarcoidosis is uncommon, but should be kept in mind even as a lone presentation. It should also be noted that in view of the ever-increasing use of MRI as an imaging modality, that this diagnosis will likely become a lot more common, as did other lesions detected on MRI. The clinical significance of these MRI findings is uncertain and has to be determined.

\section{REFERENCES}

1. Eriksen EF. Treatment of bone marrow lesions (bone marrow edema). BoneKEy Rep 2015;4:755.

2. Bauerle T, Semmler W. Imaging response to systemic therapy for bone metastases. Eur Radiol 2009;19:2495-2507.

3. O'Sullivan GJ, Carty FL, Cronin CG. Imaging of bone metastasis: an update. World J Radiol 2015;7:202-211.

4. Hanrahan CJ, Shah LM. MRI of spinal bone marrow: part 2,T1-weighted imaging-based differential diagnosis. AJR Am J Roentgenol 2011;197:1309-1321.

5. Valencia MP, Deaver PM, Mammarappallil MC. Sarcoidosis of the thoracic and lumbar vertebrae, mimicking metastasis or multifocal osteomyelitis by MRI: case report. Clin Imaging 2009;33:478-481.

6. Caobelli F, Gabanelli SV, Brucato A, Pizzocaro C, Soffientini A, Giubbini R, et al. Unsuspected active sarcoidosis diagnosed by $18 \mathrm{~F}-\mathrm{FDG}$ PET/CT during the search for a primary tumour in a patient with bone lesions. Nucl Med Mol Imaging 2013;47:205-207.

7. Lefere M, Larbi A, Malghem J, Vande Berg B, Dallaudiere B. Vertebral sarcoidosis: long-term follow-up with MRI. Skeletal Radiol 2014;43:1185-1190.

8. Rua-Figueroa I, Gantes MA, Erausquin C, Mhaidli H, Montesdeoca A. Vertebral sarcoidosis: clinical and imaging findings. Semin Arthritis Rheum 2002;31:346-352.

9. Rodman T, Funderburk EE, Jr., Myerson RM. Sarcoidosis with vertebral involvement. Ann Int Med 1959;50:213-218.

10. Johnson AK, Johnson JM, Ames E, Filippi C. Spontaneous clinical and radiological resolution of vertebral sarcoidosis: a case report. Spine 2012;37:E414-416.

11. Mehrotra P, Muller M, Higgins B, Zammit-Maempel I. An unusual cause of widespread lytic bone lesions caused by sarcoidosis. J Radiol Case Rep 2011;5:1-7.

12. Soussan M, Augier A, Brillet PY, Weinmann P, Valeyre D. Functional imaging in extrapulmonary sarcoidosis: FDG-PET/CT and MR features. Clin Nucl Med 2014;39:e146-159.

13. Mostard RL, Prompers L, Weijers RE, van Kroonenburgh MJ, Wijnen PA, Geusens PP, et al. F-18 FDG PET/CT for detecting bone and bone marrow involvement in sarcoidosis patients. Clin Nucl Med 2012;37:21-25. 